\title{
STABILITY ANALYSIS OF FUZZY CONTROL SYSTEMS. MULTIVARIABLE POINT OF VIEW
}

\author{
Radu-Emil Precup and Stefan Preitl \\ "Politehnica" University of Timisoara, Dept. of Automation and Applied Informatics \\ Bd. V. Parvan 2, RO-300223 Timisoara, Romania \\ Phone: +40-256-40-32-29, -30, -26, -24, Fax: +40-256-4032-14 \\ E-mail: rprecup@aut.utt.ro, spreitl@aut.utt.ro
}

\begin{abstract}
The paper proposes a new global stability analysis method dedicated to fuzzy control systems containing Mamdani fuzzy controllers with dynamics to control SISO linear / linearized plants. The method is expressed in terms of relatively simple steps based on the generalization of the describing function method to the MIMO case and on the application of the least squares method to approximate the describing functions. The method is applied to the stability analysis of a class of PI-fuzzy controlled servo systems and validated by a case study. Copyright (C) 2005 IFAC
\end{abstract}

Keywords: fuzzy systems, nonlinear control systems, describing functions, stability analysis, PI controllers, limit cycles.

\section{INTRODUCTION}

Fuzzy control systems (abbreviated as FCSs) are nonlinear control systems, and the dynamic behaviour of these systems is more complex than that of linear systems. With this respect the main differences between the nonlinear systems and the linear ones are: the nonlinear systems can reach multiple steady-state regimes instead of the singlepoint attractor associated with the operating point in the case of linear systems; the nonlinear systems can perform long-term behaviours including the limit cycles and the chaotic behaviours, that are more complicated than the point attractors corresponding to linear systems.

In the general framework of the qualitative theory of nonlinear dynamical systems (Khalil, 1991), some approaches and overviews (Driankov, et al., 1993; Sugeno, 1999) have been widely used to the stability analysis of FCSs. These approaches include: the state-space approach, based on a linearized model of the nonlinear dynamical system (Garcia-Cerezo and Ollero, 1992; Precup, et al., 2000); Popov's hyperstability theory (Opitz, 1993); Lyapunov's stability theory (Passino and Yurkovich, 1998;
Sugeno, 1999); the circle criterion (Driankov, et al., 1993; Passino and Yurkovich, 1998); the describing function method referred to also as the harmonic balance method (Passino and Yurkovich, 1998), etc.

The paper aim is to propose an original stability analysis method (SAM) that belongs to the fifth approach. It is dedicated to FCSs employing Mamdani fuzzy controllers with dynamics to control SISO linear/linearized plants. The support to use a fuzzy controller (FC) to a plant with linear/linearized model is: the controlled plant $(\mathrm{CP})$ model can be considered as a simplified model of a relatively complex CP. So, the CP is nonlinear but linearized in the vicinity of a set of operating points or of a trajectory. The FC, as essential nonlinear element, can compensate for the model uncertainties, the nonlinearities and the $\mathrm{CP}$ parametric variations.

The SAM proposed in the paper is based on the describing function method and represents a global SAM. Current approaches to the describing function method used in the stability analysis of FCSs deal with the following applications of this method: in the form of the exponential-input describing function technique to FCSs using the representations of the 
FCs as multidimensional-multilevel relays (Abdelnour, et al., 1993a, 1993b); to FCSs based on Mamdani FCs without dynamics (Passino and Yurkovich, 1998); to multivariable Takagi-Sugeno FCSs (Cuesta, et al., 1999); to FCSs based on FCs with dynamics, by using the describing function of the saturation which occurs outside the input universes of the FCs (Aracil and Gordillo, 2000). All these approaches require the prediction of the limit cycles, specific to the describing function method (Kim, et al., 2000).

Since the FCs with dynamics can be considered from the point of view of the basic fuzzy controller (without dynamics) as MIMO systems, the main contribution of this paper is to generalize the describing function method to the MIMO case of FCSs based on Mamdani FCs with dynamics. The application of the least squares method enables the approximation of the describing functions. The proposed SAM is expressed in terms of transparent and relatively simple steps.

This paper is organized as follows. The next Section deals with the presentation of the considered class of Mamdani FCs with dynamics focussed on the PIfuzzy controllers with output integration. In Section 3 the describing function method is applied, the resulting SAM is derived and the SAM steps are presented. Then, Section 4 addresses the application of the SAM to the stability analysis of a class of FCSs to control a class of servo systems and validates the proposed method by considering a case study. Section 5 draws the conclusions.

\section{A CLASS OF MAMDANI FUZZY CONTROLLERS WITH DYNAMICS}

The structure of the considered FCS is a conventional one, presented in Fig. 1, where: $r$ - the reference input, $y-$ the controlled output, $e=r-y$ - the control error, $u$ - the control signal, $d_{1}, d_{2}, d_{3}-$ the disturbance inputs, and the $\mathrm{CP}$ includes the actuator and the measuring device.

The PI-fuzzy controller with output integration (PIFC-OI) represents the core of the considered class of Mamdani FCs with dynamics and singleton consequents, part of the general class of type-II fuzzy systems (Sugeno, 1999). The PI-FC-OI is a discretetime FC with dynamics, introduced by the numerical differentiation of the control error $e_{k}$ expressed as the increment of control error, $\Delta e_{k}, \Delta e_{k}=e_{k}-e_{k-1}$, and by the numerical integration of the increment of control signal, $\Delta u_{k}, u_{k}=u_{k-1}+\Delta u_{k}$, with $k-$ index of the current sampling interval. The PI-FC-OI structure is shown in Fig. 2, where B-FC represents the basic fuzzy controller, without dynamics.

Since many PID controllers can be brought to the series form with the transfer function (t.f.) $H_{C}(s)$ :

$$
H_{C}(s)=k_{c}\left(1+s T_{c 1}\right)\left(1+s T_{c 2}\right) / s,
$$

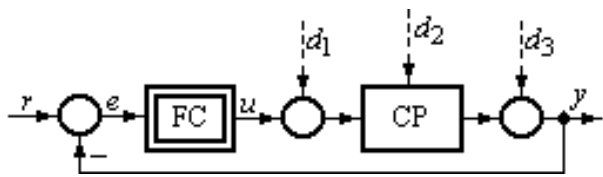

Fig. 1. Structure of fuzzy control system.

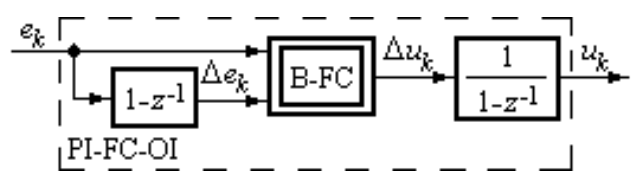

Fig. 2. Structure of PI-fuzzy controller with output integration.

where $k_{c}$ is the controller gain and $T_{c 1}, T_{c 2}$ are the controller time constants, the FC structure shown in Fig. 2 can be used also in the situations of PD-fuzzy controllers, by omitting the integral block in Fig. 2, or of PID-fuzzy controllers, by adding a series connection PD term after the integral block in Fig. 2. This PD term can be seen in specific situations to belong to the linear part of the CP to simplify the SAM. The application of the PI-FC-OI structure to the PID-FCs is valid only when the $\mathrm{CP}$ parameters are time-varying but relatively slowly. In such situations the use of more complex FCs (for example, with three inputs), although possible, is not justified.

The block B-FC is a nonlinear two inputs-single output (TISO) system, which includes among its nonlinearities the scaling of inputs and output as part of its fuzzification module. The fuzzification is solved in the initial phase by means of the membership functions (m.f.s) in Fig. 3. Other m.f.s distributions can modify in a desired way the B-FC nonlinearities (Babuška and Verbruggen, 1996). The inference engine in B-FC employs Mamdani's MAX-MIN compositional rule of inference assisted by the rule base in Table 1, and the centre of gravity method for singletons is used for defuzzification. Fig. 3 points out the three strictly positive parameters of the PI-FC-OI to be tuned by an accordingly applied development method, $B_{e}, B_{\Delta e}$ and $B_{\Delta u}$.

The start in the PI-FC-OI development is to express the discrete-time equation of a digital PI controller:

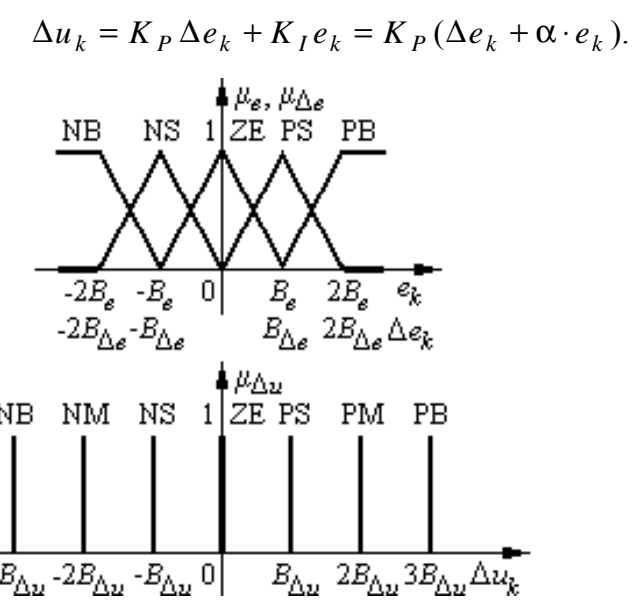

Fig. 3. Membership functions of input and output linguistic variables in B-FC. 
Table 1 Decision table of B-FC

\begin{tabular}{llllll}
\hline$\Delta e_{k} \backslash e_{k}$ & NB & NS & ZE & PS & PB \\
\hline PB & ZE & PS & PM & PB & PB \\
PS & NS & ZE & PS & PM & PB \\
ZE & NM & NS & ZE & PS & PM \\
NS & NB & NM & NS & ZE & PS \\
NB & NB & NB & NM & NS & ZE \\
\hline
\end{tabular}

In the case of a quasi-continuous digital PI controller the parameters $K_{P}, K_{I}$ and $\alpha$ can be computed as functions of the parameters $k_{C}$ (gain) and $T_{i}$ (integral time constant) of a basic continuous-time PI controller having the t.f. $H_{C}(s)$ :

$$
H_{C}(s)=k_{C}\left(1+1 /\left(s T_{i}\right)\right),
$$

and the connections between $\left\{K_{P}, K_{I}, \alpha\right\}$ and $\left\{k_{C}, T_{i}\right\}$ are (4) in the case of Tustin's method:

$$
\begin{aligned}
& K_{P}=k_{C}\left[1-T_{s} /\left(2 T_{i}\right)\right], K_{I}=k_{C} T_{s} / T_{i}, \\
& \alpha=K_{I} / K_{P}=2 T_{s} /\left(2 T_{i}-T_{s}\right),
\end{aligned}
$$

with $T_{s}$ - sampling period chosen in accordance with the requirements of quasi-continuous digital control.

The development equations of the PI-FC-OI are obtained by the modal equivalence principle:

$$
B_{\Delta e}=\alpha \cdot B_{e}, B_{\Delta u}=K_{I} B_{e},
$$

where the free parameter $B_{e}$ represents the designer's option. Using the experience in controlling the plant one can choose the value of this parameter, but firstly it must be chosen to ensure the aim of a stable FCS.

\section{DESCRIBING FUNCTION METHOD}

To formulate the SAM based on the application of the describing function method it is necessary to transform the initial FCS structure into a MIMO one because the block B-FC in Fig. 2 is a TISO system. This modified FCS structure is illustrated in Fig. 4, where the dynamics of the fuzzy controller (its linearized part) is transferred to the $\mathrm{CP}$. The result will be the extended controlled plant (ECP, a linear block). The vectors in Fig. 4, $\mathbf{r}_{k}$ - the reference input vector, $\mathbf{e}_{k}-$ the control error vector, $\mathbf{u}_{k}-$ the control signal vector and $\mathbf{y}_{k}$ - the controlled output vector, are defined as follows:

$$
\begin{aligned}
& \mathbf{r}_{k}=\left[\begin{array}{ll}
r_{k} & \Delta r_{k}
\end{array}\right]^{T}, \mathbf{e}_{k}=\left[\begin{array}{ll}
e_{k} & \Delta e_{k}
\end{array}\right]^{T}, \\
& \mathbf{u}_{k}=\left[\begin{array}{ll}
\Delta u_{k} & \Delta u_{f, k}
\end{array}\right]^{T}, \mathbf{y}_{k}=\left[\begin{array}{ll}
y_{k} & \Delta y_{k}
\end{array}\right]^{T},
\end{aligned}
$$

where: $\Delta \mathbf{v}_{k}=\mathbf{v}_{k}-\mathbf{v}_{k-1}-$ the increment of the vector $\mathbf{v}_{k}, \Delta u_{f, k}$ - the fictitious increment of control signal, $T$ - index used in matrix transposition. The FCS structure in Fig. 4 can be used in both the discrete-time case and the continuous-time case, with the vectors defined in terms of (7) (connected to (6)):

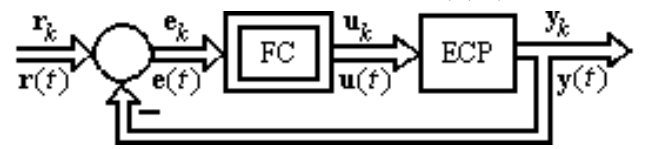

Fig. 4. Structure of FCS used in stability analysis.

$$
\begin{aligned}
& \mathbf{r}(t)=\left[\begin{array}{ll}
w_{1}(t) & w_{2}(t)
\end{array}\right]^{T}, \mathbf{e}(t)=\left[\begin{array}{ll}
i_{1}(t) & i_{2}(t)
\end{array}\right]^{T}, \\
& \mathbf{u}(t)=\left[\begin{array}{ll}
c_{1}(t) & c_{2}(t)
\end{array}\right]^{T}, \mathbf{y}(t)=\left[\begin{array}{ll}
o_{1}(t) & o_{2}(t)
\end{array}\right]^{T} .
\end{aligned}
$$

The nonlinear input-output static map $\mathbf{F}$ characterizes the block FC in Fig. 4:

$$
\mathbf{F}: R^{2} \rightarrow R^{2}, \mathbf{F}\left(\mathbf{e}_{k}\right)=\left[f\left(\mathbf{e}_{k}\right), 0\right]^{T},
$$

where $f\left(f: R^{2} \rightarrow R\right)$ is the input-output static map of the nonlinear TISO system B-FC in Fig. 2. Therefore, the modified FCS structure presented in Fig. 4 has only one time-invariant and memoryless nonlinear component, the block FC. In this context, to apply the describing function method two conditions must be fulfilled (Passino and Yurkovich, 1998): the nonlinear function $\mathbf{F}$ should be an odd function, and the block ECP should have characteristics of a low-pass filter.

All vectors in the modified FCS structure have two components (see (6) and (7)). This required the introduction of a fictitious control signal, supplementary to the outputs of the block B-FC, to obtain an equal number of inputs and outputs, necessary to the stability theory in the MIMO case.

The mathematical model of the block ECP can be derived by starting with the t.f. of the $\mathrm{CP}$ with respect to the control signal, $H_{C P}(s)$. Discretizing $H_{C P}(s)$ by taking into account the presence of a zeroorder hold block - in the conditions of $T_{s}$ chosen in accordance with the requirements of quasicontinuous digital control - leads to the pulse t.f. of the controlled plant, $H_{Z C P}(z)$ :

$$
H_{Z C P}(z)=\left(1-z^{-1}\right) Z\left\{L^{-1}\left[H_{C P}(s) / s\right]\right\} .
$$

The pulse t.f. of the CP together with the integral block (in Fig. 2), $H_{I C P}(z)$, can be expressed as:

$$
H_{I C P}(z)=Z\left\{L^{-1}\left[H_{C P}(s) / s\right]\right\} .
$$

To obtain the continuous-time equivalents $H_{11}(s)$ and $H_{21}(s)$ of the pulse t.f.s in (10) and (9), respectively, Tustin's method mapping the z-plane to the s-plane:

$$
\left[\begin{array}{l}
H_{11}(s) \\
H_{21}(s)
\end{array}\right]=\left.\left[\begin{array}{l}
H_{I C P}(z) \\
H_{Z C P}(z)
\end{array}\right]\right|_{z=\frac{1+T_{s} / 2}{1-T_{s} / 2}} .
$$

By using the definition of the vectors in (7), the transfer matrix $\mathbf{H}(s)$ of the block ECP will be (12):

$$
\mathbf{H}(s)=\left[H_{i j}(s)\right]_{i, j=\overline{1,2}} .
$$

The two t.f.s $H_{12}(s)$ and $H_{22}(s)$ in (12) have no effect on the SAM because they correspond to the fictitious control signal, $c_{2}(t)$ (in the discrete case, to its fictitious increment, $\left.\Delta u_{f, k}\right)$. This is the reason for the zero in (8). Feeding an input $\mathbf{e}(t)$ to FC (in Fig. 4):

$$
i_{l}(t)=A_{l} \sin (\omega t), l=\overline{1,2},
$$

where the input amplitudes are $\mathbf{a}=\left[\begin{array}{ll}A_{1} & A_{2}\end{array}\right]^{\mathrm{T}}, A_{l}>0$, $l=\overline{1,2}$, and the frequency is $\omega>0$, will result in the output $\mathbf{u}(t)$ expanded into a Fourier series: 


$$
\begin{aligned}
c_{m}(t) & =a_{0, m} / 2+\sum_{n=1}^{\infty}\left[a_{n, m} \cos (n \omega t)+\right. \\
& \left.+b_{n, m} \sin (n \omega t)\right], \quad m=\overline{1,2},
\end{aligned}
$$

with the expressions (15) of the Fourier coefficients:

$$
\begin{aligned}
& a_{0, m}=(1 / \pi) \int_{-\pi}^{\pi} c_{m}(t) d(\omega t), a_{n, m}+j b_{n, m}= \\
& =(1 / \pi) \int_{-\pi}^{\pi} c_{m}(t) e^{j \omega t} d(\omega t), n \in N^{*}, m=\overline{1,2} .
\end{aligned}
$$

In the accepted conditions, $a_{0, m}=0$ and by neglecting the higher order harmonics with respect to the fundamental harmonics, the result will be expressed as the describing functions $N_{m l}(\mathbf{a}, \omega)$ that will not depend on $\omega$ and so will be written as $N_{m l}(\mathbf{a})$ :

$$
N_{m l}(\mathbf{a})=b_{1, m} / A_{l}, l, m=\overline{1,2} .
$$

Neglecting the effect of the fictitious (increment of) control signal on the SAM requires the necessity for:

$$
N_{2 l}(\mathbf{a})=0, l=\overline{1,2} .
$$

Defining the describing function matrix $\mathbf{N}(\mathbf{a})$ :

$$
\mathbf{N}(\mathbf{a})=\left[N_{m l}(\mathbf{a})\right]_{l, m=1,2},
$$

the harmonic balance equation will be written as (Cuesta, et al., 1999):

$$
(\mathbf{H}(j \omega) \mathbf{N}(\mathbf{a})+\mathbf{I}) \mathbf{a}=\left[\begin{array}{ll}
0 & 0
\end{array}\right]^{T}, \mathbf{I}=\operatorname{diag}(1,1) .
$$

If any limit cycles exist in the FCS, then their existence can be predicted by solving the equation (19) with respect to a and $\omega$.

There can be employed several ways to compute the describing functions. This problem can be solved easily if the analytical expression of the function $f$ is known. If this is not the case, the problem is more complex and can be solved by using the numerical integration in (15). Another approach, to be presented as follows, is based on performing an experiment with the nonlinear block FC in Fig. 4, assisted by the application of the least squares method. This approach to compute the describing function matrix consists of the following steps:

- step 1: excite the inputs of the block FC with the sinusoidal signals (obtained in (13)) $i_{l}(t)$ :

$$
i_{l}(t)=A_{l}^{q} \sin (\omega t), l=\overline{1,2}, q=\overline{1, M},
$$

$i_{h}(t)=0$ if $h \neq l$, for a certain value of $\omega>0$, and a set of $M$ values of amplitudes $A_{l}^{q}>0$;

- step 2: wait for the FC to enter an oscillatory permanent regime and save the values of the $q$ output functions $c_{1}$, written as $c_{1}^{q}$; these values can be approximated analytically by the expressions (21):

$$
\begin{aligned}
& c_{1}^{q}\left(t_{i}\right) \approx a_{1, l}^{q} \cos \left(\omega t_{i}\right)+b_{1, l}^{q} \sin \left(\omega t_{i}\right), \\
& l=\overline{1,2}, q=\overline{1, M}, i=\overline{1, P},
\end{aligned}
$$

with $P$ - the number of time moments considered, $t_{i}$;
- step 3: introduce the matrices $\mathbf{y}_{1}^{q}, \mathbf{S}^{q}, \mathbf{p}_{l}^{q}$ :

$$
\begin{aligned}
& \mathbf{y}_{1}^{q}=\left[\begin{array}{c}
c_{1}^{q}\left(t_{1}\right) \\
c_{1}^{q}\left(t_{2}\right) \\
\ldots \\
c_{1}^{q}\left(t_{P}\right)
\end{array}\right], \mathbf{S}^{q}=\left[\begin{array}{cc}
\cos \left(\omega t_{1}\right) & \sin \left(\omega t_{1}\right) \\
\cos \left(\omega t_{2}\right) & \sin \left(\omega t_{2}\right) \\
\ldots & \\
\cos \left(\omega t_{P}\right) & \sin \left(\omega t_{P}\right)
\end{array}\right], \\
& \mathbf{p}_{l}^{q}=\left[\begin{array}{c}
a_{1, l}^{q} \\
b_{1, l}^{q}
\end{array}\right], l=\overline{1,2}, q=\overline{1, M},
\end{aligned}
$$

corresponding to the two objective functions $J_{l}^{q}$ :

$$
\begin{aligned}
J_{l}^{q} & =\sum_{i=1}^{P}\left[c_{1}^{q}\left(t_{i}\right)-a_{1, l}^{q} \cos \left(\omega t_{i}\right)-\right. \\
& \left.-b_{1, l}^{q} \sin \left(\omega t_{i}\right)\right], l=\overline{1,2}, q=\overline{1, M},
\end{aligned}
$$

and the minimization of $J_{l}^{q}$ with respect to $\mathbf{p}_{l}^{q}$ leads to the solutions of the least squares method:

$$
\mathbf{p}_{l}^{q}=\left[\left(\mathbf{S}^{q}\right)^{T} \mathbf{S}^{q}\right]^{-1}\left(\mathbf{S}^{q}\right)^{T} \mathbf{y}_{1}^{q},
$$

where only the second components of $\mathbf{p}_{l}^{q}$ are of interest, $b_{1, l}^{q}$, and $\left[\left(\mathbf{S}^{q}\right)^{T} \mathbf{S}^{q}\right]$ in (24) should be regular (requiring the proper choice of the time moments, $t_{i}$ ); - step 4: the values of the describing functions will be obtained in terms of the following equations obtained from (16) and (17):

$$
N_{1 l}\left(\mathbf{a}^{q}\right)=b_{1, l}^{q} / A_{l}^{q}, N_{2 l}\left(\mathbf{a}^{q}\right)=0, l=\overline{1,2},
$$

where $\mathbf{a}^{q}=\left[\begin{array}{ll}A_{1}^{q} & A_{2}^{q}\end{array}\right]^{T}, q=\overline{1, M}$.

By taking into account all aspects presented before, the SAM can be formulated in terms of the following steps:

- step (a): express the transfer matrix of the block ECP in (12) by using (9) ... (11);

- step (b): compute $M$ values of the describing function matrix $\mathbf{N}\left(\mathbf{a}^{q}\right), q=\overline{1, M}$, in terms of the steps 1 ... 4;

- step (c): obtain the solutions of the harmonic balance equation (19) by using numerical techniques to solve the optimization problem (26):

$$
\min _{\mathbf{a} \in R^{2}, \omega>0}\|(\mathbf{H}(j \omega) \mathbf{N}(\mathbf{a})+\mathbf{I}) \mathbf{a}\|,
$$

having the solutions $\mathbf{a}^{*}-$ the input amplitudes and $\omega^{*}$ - the frequency of the limit cycles, and $\|\mathbf{v}\|$ represents generally the Euclidean norm of the vector $\mathbf{v}$; - step (d): solve the optimization problem (27):

$$
\min _{\mathbf{a} \in R^{2}, \omega>0}\left\|(\mathbf{H}(j \omega) \mathbf{N}(\mathbf{a})+\mathbf{I}) \mathbf{a}-\boldsymbol{\varepsilon}_{i}\right\|,
$$

for two relatively small values of $\boldsymbol{\varepsilon}_{i}=\left[\begin{array}{ll}\varepsilon_{1, i} & \varepsilon_{2, i}\end{array}\right]^{T}$, $i=\overline{1,2}, \varepsilon_{1,1}>0, \varepsilon_{2,1}>0, \varepsilon_{1,2}<0, \varepsilon_{2,2}<0$, and the solutions of (27) are $\mathbf{a}_{i}$ and $\omega_{i}, i=\overline{1,2}$;

- step (e): if $\left\|\mathbf{a}_{1}\right\|<\left\|\mathbf{a}^{*}\right\|$ and $\left\|\mathbf{a}_{2}\right\|>\left\|\mathbf{a}^{*}\right\|$, then the corresponding limit cycle will be stable and the FCS will be globally stable. The FCS is globally stable also if the equation (26) does not have any solutions. 


\section{APPLICATION}

This Section is dedicated to the validation of the proposed SAM by its application to the stability analysis of a class of FCSs to control a class of servo systems, where the CP has the simplified t.f. (28):

$$
H_{C P}(s)=k_{P} /\left[\left(1+s T_{\Sigma}\right)\left(1+s T_{1}\right)\right],
$$

where: $T_{1}-$ large time constant, $T_{\Sigma}-$ small time constant or time constant corresponding to the sum of parasitic time constants $\left(T_{\Sigma}<<T_{1}\right)$, and $k_{P}-$ gain. In these conditions, from a theoretical point of view, $H_{C P}(s)$ has a quasi-integral behaviour, and the benchmark (29) can be used to approximate (28):

$$
H_{C P}(s)=k_{P} /\left[s\left(1+s T_{\Sigma}\right)\right] .
$$

For the CP (29) the use of PI controllers having the t.f. (3) or (30) used as basic continuous-time PI controllers in accordance with Section 2:

$$
H_{C}(s)=k_{c}\left(1+s T_{i}\right) / s, k_{c}=k_{C} / T_{i},
$$

with the gain $k_{c}$ tuned in terms of the Extended Symmetrical Optimum (ESO) method (Preitl and Precup, 1999), can ensure good control system (CS) performance:

$$
k_{c}=1 /\left(\beta^{3 / 2} T_{\Sigma}^{2} k_{P}\right), T_{i}=\beta T_{\Sigma}, k_{C}=k_{c} T_{i},
$$

where $\beta$ represents a single design parameter.

The tuning relations (31) were obtained by applying the optimization conditions (32):

$$
\beta^{1 / 2} a_{0} a_{2}=a_{1}^{2}, \beta^{1 / 2} a_{1} a_{3}=a_{2}^{2},
$$

specific to the ESO method, in the case of the closedloop t.f. with respect to the reference input $H_{r}(s)$ (the $\mathrm{CS}$ structure is that in Fig. 1, with the continuoustime PI controller instead of the block FC):

$$
\begin{aligned}
H_{r}(s) & =\left(b_{0}+b_{1} s\right) /\left(a_{0}+a_{1} s+a_{2} s^{2}+\right. \\
& \left.+a_{3} s^{3}\right), b_{0}=a_{0}, b_{1}=a_{1} .
\end{aligned}
$$

The ESO method represents a generalization of the Symmetrical Optimum method (Åström and Hägglund, 1995), and it performs indeed an optimization since it guarantees the maximum phase margin $\varphi_{m}$ for constant $\mathrm{CP}$ parameters and a minimum $\varphi_{m}$ for variable $k_{P}$. The CS performance indices $\left\{\sigma_{1}-\right.$ overshoot, $\hat{t}_{s}=t_{s} / T_{\Sigma}-$ settling time, $\hat{t}_{r}=t_{r} / T_{\Sigma}$ - rise time, $\left.\varphi_{m}\right\}$ can be modified by the choice of the $\beta$ in the recommended domain, $1<\beta<20$. A compromise to these indices can be reached by using the diagrams illustrated in Fig. 5. The CS performance indices can be improved by adding feed-forward filters (Preitl and Precup, 1999).

In the case of the controlled plant (28) and of the PI controller with the t.f. (30), the coefficients of $H_{r}(s)$ in (33) can be expressed in terms of (34):

$$
a_{0}=k_{c} k_{P}, a_{1}=k_{c} k_{P} T_{1}, a_{2}=T_{1}+T_{\Sigma}, a_{3}=T_{1} T_{\Sigma} .
$$

Applying the optimization conditions (32) and introducing the notation (35) to characterize the CP:

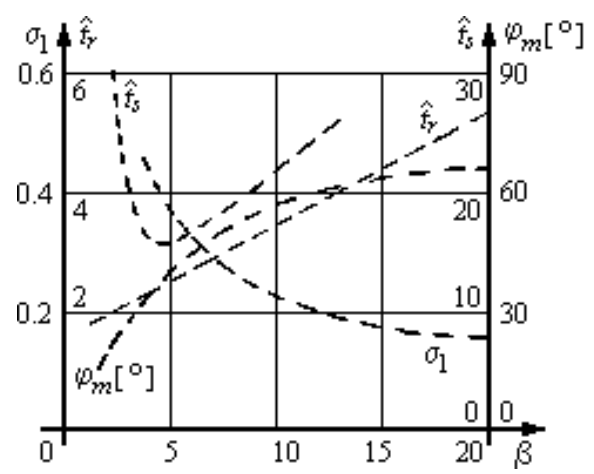

Fig. 5. Control system performance indices versus $\beta$.

$$
m_{P}=T_{\Sigma} / T_{1}<<1,
$$

the tuning relations will obtain the form (36) in this case, corresponding to an extension of the ESO method (Preitl, et al., 2002):

$$
\begin{aligned}
& k_{c}=\left(1+m_{P}\right)^{3} /\left(\beta^{3 / 2} T_{\Sigma} k_{P} m_{P}\right), T_{i}=\beta T_{\Sigma m}, \\
& T_{\Sigma m}=T_{\Sigma}\left[m_{P}^{2}+\left(2-2^{1 / 2}\right) m_{P}+1\right] /\left(1+m_{P}\right)^{3} .
\end{aligned}
$$

The recommended values for $\beta$ are again $1<\beta<20$.

The considered case study can correspond to relatively complex servo systems plants having mathematical models that can be transformed in the simplified t.f. (28), with the parameters $k_{P}=1, T_{\Sigma}=1 \mathrm{~s}$ and $T_{1}=10 \mathrm{~s}$, fulfilling (35) because $m_{P}=0.1$. The development of the PI-FC-OI is done according to Section 2, beginning with the development of the basic continuous-time PI controller in terms of this Section. Choosing $\beta=9$ and applying (36), the PI controller parameters will have the values $k_{c}=0.493$, $T_{i}=7.2256 \mathrm{~s}, k_{C}=3.5619$. Setting $T_{s}=0.5 \mathrm{~s}$, the parameters of the quasi-continuous digital PI controller result from (4): $K_{P}=3.5273, K_{I}=0.2465$, $\alpha=0.0699$. The choice of the PI-FC-OI parameter $B_{e}$ $=0.5$ and (5) will lead to the other two PI-FC-OI parameters, $B_{\Delta e}=0.0349$ and $B_{\Delta u}=0.1232$. In these conditions, there will be applied as follows the steps (a) ... (e) of the SAM according Section 3:

- step (a): the useful elements of $\mathbf{H}(s)$ are:

$$
\begin{aligned}
& H_{11}(s)=B_{11}(\mathrm{~s}) / A_{11}(\mathrm{~s}), B_{11}(\mathrm{~s})=0.0003 \mathrm{~s}^{3}+ \\
& +0.0145 s^{2}+0.1024 s+0.1959 \\
& A_{11}(\mathrm{~s})=\mathrm{s}\left(\mathrm{s}^{2}+1.0797 s+0.0979\right) \\
& H_{21}(s)=B_{21}(\mathrm{~s}) / A_{21}(\mathrm{~s}) \\
& B_{21}(\mathrm{~s})=0.0006 \mathrm{~s}^{2}+0.02767 s+0.0979 \\
& A_{21}(\mathrm{~s})=\mathrm{s}^{2}+1.0797 s+0.0979
\end{aligned}
$$

- step (b): due to the distribution of the m.f.s (Fig. 3) and to the defuzzification method, the PI-FC-OI will have quasi-PI behaviour (that means it will be almost linear), and it is sufficient a relatively small number of values of the describing function matrix, $M=10$. In the case $\omega=1 \mathrm{rad} / \mathrm{s}$, the elements of $\mathbf{N}\left(\mathbf{a}^{q}\right)$ are:

$$
\begin{aligned}
& \mathbf{a}^{q} \in\{0.1,0.2,0.4,0.5,0.6,0.8,1,1.1, \\
& 1.2,1.5\}, N_{11}^{q}=N_{12}^{q} \in\{0.308,0.232,0.2377, \\
& 0.2332,0.2468,0.246,0.2437,0.2408, \\
& 0.2307,0.2437\}, N_{21}^{q}=N_{22}^{q}=0, q=\overline{1,10} .
\end{aligned}
$$


Some elements in (38) are equal one to another because the nonlinearity of B-FC is symmetrical (caused by the m.f.s shapes in Fig. 3 and by the decision table). Other elements in (38) are zero to neglect the effect of $\Delta u_{f, k}$;

- step (c): the equation (26) does not have any solutions, which ensures the global stability of the FCS in the case $B_{e}=0.5$. This is the reason why the steps (d) and (e) are no more necessary.

The behaviour of the developed FCS is illustrated in Fig. 6 in the simulation conditions characterized by the unit step modification of $r$ followed by a -0.5 step modification of the disturbance input $d_{3}$ (after 75 s), where the continuous line is used for $y$ and the dotted line for $u$. This behaviour proves that the FCS is globally stable for the developed FC.

\section{CONCLUSIONS}

The paper offers a new SAM representing a generalization to the MIMO case of other limit cycle criteria. The SAM enables the redesign of the fuzzy controllers to avoid the existence of limit cycles, and this method can be considered as part of a development method for Mamdani fuzzy controllers with dynamics.

The proposed SAM is expressed in a transparent manner by steps that provide useful information to the choice of the free parameters of the FCs $\left(B_{e}\right.$ in the case of the PI-FC-OI).

The application presented in the paper validates the SAM and opens perspectives to apply it to FCSs employing other fuzzy controllers with dynamics including the PD- and PID-FCs.

Although the SAM derives sufficient stability conditions, for the considered case study these conditions are stronger in comparison with the first four approaches mentioned in Section 1 that do not guarantee that the FCS developed here is globally stable.

The plant considered in this paper has been assumed linear. It must be seen as a linearized one, corresponding to a more complex model of a servo system. This is valid because generally the goal of fuzzy control is not to control simple plants, but to initially approach by a convenient and understandable nonlinear solution the control of a complex, uncertain or not well-defined plant.

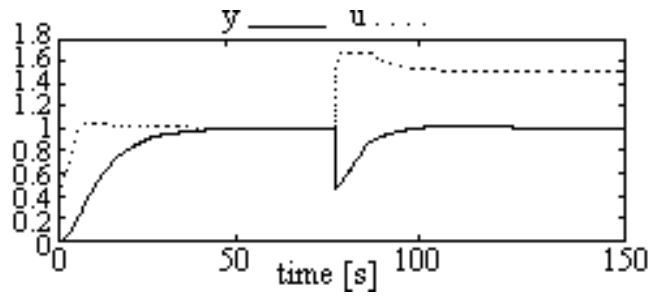

Fig. 6. Behaviour of fuzzy control system.

\section{REFERENCES}

Abdelnour, G., J.Y. Cheung, C.-H. Chang and G. Tinetti (1993a). Application of describing functions in the transient response analysis of a three-term fuzzy controller. IEEE Trans. Systems, Man, and Cybernetics, 23, 603-606.

Abdelnour, G., J.Y. Cheung, C.-H. Chang and G. Tinetti (1993b). Steady-state analysis of a threeterm fuzzy controller. IEEE Trans. Systems, Man, and Cybernetics, 23, 607-610.

Aracil, J. and F. Gordillo (2000). Describing function method for stability Analysis of PD and PI fuzzy controllers. Preprints of IFAC Workshop on Digital Control: Past, Present and Future of PID Control-PID'00, Terrassa, Spain, 599-605.

Åström, K.J. and T. Hägglund (1995). PID Controllers Theory: Design and Tuning. Instrument Society of America, Research Triangle Park, NC.

Babuška, R. and H.B. Verbruggen (1996). An Overview on Fuzzy Modeling for Control. Control Engineering Practice, 4, 1593-1606.

Cuesta, F., F. Gordillo, J. Aracil and A. Ollero (1999). Stability analysis of nonlinear multivariable Takagi-Sugeno fuzzy control systems. IEEE Trans. Fuzzy Systems, 7, 508520.

Driankov, D., H. Hellendoorn and M. Reinfrank (1993). An Introduction to Fuzzy Control. Springer-Verlag, Berlin, Heidelberg, New York.

Garcia-Cerezo, A. and A. Ollero (1992). Stability of fuzzy control systems by using nonlinear system theory. Proceedings of IFAC / IFIP / IMACS Symp. on Artificial Intelligence in Real-Time Control, Delft, Netherlands, 171-176.

Kim, E., H. Lee and M. Park (2000). Limit-cycle prediction of a fuzzy control system based on describing function method. IEEE Trans. Fuzzy Systems, 8, 11-22.

Opitz, H.-P. (1993). Fuzzy control and stability criteria. Proc. of $1^{\text {st }}$ European Congress on Fuzzy and Intelligent Technologies-EUFIT'93, Aachen, Germany, 1, 130-136.

Passino, K.M. and S. Yurkovich (1998). Fuzzy Control. Addison-Wesley, Menlo Park, CA.

Precup, R.-E., S. Doboli and S. Preitl (2000). Stability analysis and development of a class of fuzzy control systems. Engineering Applications of Artificial Intelligence, 13, 237-247.

Preitl, S. and R.-E. Precup (1999). An extension of tuning relations after Symmetrical Optimum method for PI and PID controllers. Automatica, 35, 1731-1736.

Preitl, S., Z. Preitl and R.-E. Precup (2002). Low cost fuzzy controllers for classes of second-order systems. Preprints of $15^{\text {th }}$ IFAC World Congress, Barcelona, Spain, paper 416, 6 pp.

Slotine, J.-J.E. and W. Li (1991). Applied Nonlinear Control. Prentice-Hall, Englewood Cliffs, NJ.

Sugeno, M. (1999). On stability of fuzzy systems expressed by fuzzy rules with singleton consequents. IEEE Trans. Fuzzy Systems, 7, 201-224. 